\title{
The architecture admin IOT in monitoring automation applied to crops hydroponic of the type N.F.T
}

\begin{abstract}
The objective of this study was to use the Admin IoT architecture for automation of monitoring hydroponic crops of type N. F. T., using API, middleware and coordinator to introduce automation in the prototype format. The experiment found that its operation is of great importance and support in automation, as well as giving comfort to the owner of crops, covers a great ease in managing their components and crop development. In addition to ease, the software proved to be safe and able to be used as actual product, moreover, is ready to be used in small and medium scale, being economically useful and easily accessible.
\end{abstract}

Volume 5 Issue I - 2019

\author{
Francisco Jose de Araujo, Raimundo Pereira \\ da Cunha Neto,Artur Felipe da Silva veloso, \\ Arleide Ribeiro Braz, Plácido das Chagas \\ Soares Segundo \\ Faculty of Engineering, Coordination of the Computer Science \\ Course, Brazil
}

Correspondence: Raimundo Pereira da Cunha Neto, Faculty of Engineering, Coordination of the Computer Science Course, Av.dos Expedicionários, 790-São João, Teresina-PI, Brazil, 64046700,Brazil, Email netocunhathe@gmail.com

Received: December 01, 2018 | Published: January 23, 2019

\section{Introduction}

The term automation is gaining strength and greater meaning to people over the years, all thanks to the technological advances that we are experiencing, and with it the increasing popularization of the Internet, where billions of people can communicate instantaneously from any on the planet. With the almost unlimited possibility that the Internet and the technological advances gives us the concept of automation is strengthened. The term automation is directly linked to the word "automatic", which refers to the execution of any task without the need for human intervention. Both the industry and the other sectors have the need to improve and accelerate their tasks effectively, and thus can have a productivity gain. With that increasingly there is a need to automate your tasks reducing spending on labor and even waste of raw materials, significantly increasing productivity. With population growth comes the need for increased food production, to meet all the demand, which raises concerns about the impact that is caused to the environment. An alternative that has been used is hydroponics. According to Graves ${ }^{1,2}$ Resh $^{3}$ Hydroponics is a term obtained by combining two words of Greek origin-hydro, meaning water and ponos, work-which is spreading rapidly to production mode vegetables, especially vegetables grown in controlled environments. Hydroponics is an alternative method of cultivation in controlled environments, which eliminates the use of the soil, thus giving rise to a solution containing only water and essential nutrients to plants.

When we talk about automation many people think of a subject that has been much discussed around the world, the Internet of Things. The term Internet of Things, named by Ashton ${ }^{4}$ became popular mainly for its success in the use of RFID technology in tracking areas of objects, people and animals. ${ }^{5}$ Thus, it is expected to pass the Internet to communicate not only people with people but also objects with people and objects with objects, Machine to Machine (M2M). Thinking about this entire evolutionary context, and the needs increasingly to facilitate the tasks of day-to-day, was developed Admin IoT architecture, which aims to represent internet architecture of things quickly, easily and safe. Thus enabling the different areas of professional easy handling and easy understanding of an automation connected to internet of things quickly and viably.

\section{Architecture}

The extensibility and transparency for any device causes the middleware covering wide variety of things beneath it. ${ }^{6}$ Thereby making the service provided for external applications, middleware above, uniform and standardized. Adding transparent APIs to middleware, applications may be written, regardless of their platform, to use the devices to understand the context in which they live and perform the appropriate action, thus bringing pervasiveness to the system. $^{7}$

The Admin IoT architecture is based on the principle in a star topology connection, mobile devices via app, website or middleware API, where is all the database information and services available for interconnectivity between them where they are connected. Through the middleware, laptops or microprocessors as the Raspberry Pi are connected to API, they are also connected to the Coordinators, i.e microcontrollers like the Arduino, which are connected to it via USB, thus making communication between them. In turn, the coordinators are connected to the other End Devices via wireless communication such as Wi-Fi, Bluetooth or radio frequency, as with exceed, such communication is made in the topology mesh format. All called for thing, will be plugged to any end device or even the coordinator.

Finally, users can access information about their things via app or website, doing so only a login with the same registration made in the API. Below is a picture showing how all Admin IoT architecture: The architecture shows how will the operation of the automation in the current concept of Internet of things (Figure 1). For this project this work will be a simpler connection, which will create a registration API, and automation in prototype format will be made entirely in the Coordinator, i.e, only in a microcontroller that is connected to the Raspberry Pi, where it will be running the middleware.

\section{Hydroponics}

Hydroponics consists of a cultivation technique without the use of soil, wherein the nutrient demand required for the normal development of the crop is supplied by providing an aqueous solution that contains all the necessary nutrients, which is commonly known 
as nutrient solution. By allowing greater control over the conditions of cultivation, hydroponic technology has the advantage of minimizing some of the problems related to the prevailing production models, such as the reduction in water waste, energy, inputs, among others. ${ }^{8}$ Douglas $^{9}$ comprises the nutrient solution is, in hydroponics, the most important factor, it replaces one of the most important functions of soil, which is the supply of nutrients for the development of plant production, thus playing an essential activity in determining the quality and productivity.

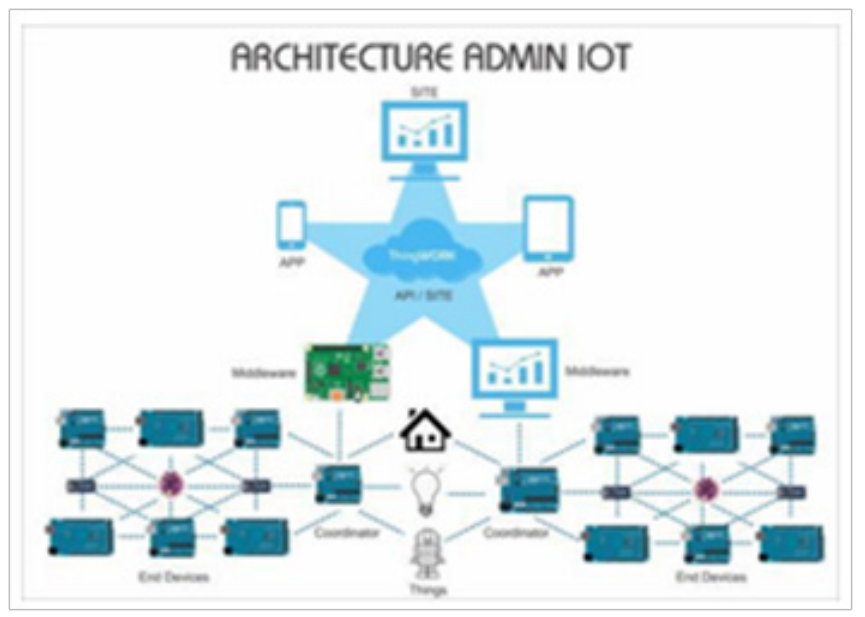

Figure I Internet of things.

Opting for hydroponics as a method for cultivation, the environmental impacts are extremely lower than the conventional methods, with the chief among them is the water because the water used is recycled thus allowing a huge reduction in the consumption of these very important resources for the planet. ${ }^{10}$ The nutrient film technique (NFT) was developed during the late 1960's by Dr Allan Cooper at the Glasshouse Crops Research Institute in the U.K. With the NFT system, a thin film of nutrient solution flows through plastic channels, which contain the plant roots with no solid planting media. The root mat develops partly in the shallow stream of recirculating solution and partly above it. It is extremely important to maintain this basic principle of a nutrient film because it ensures the root system has access to adequate oxygen levels (Figure 2).

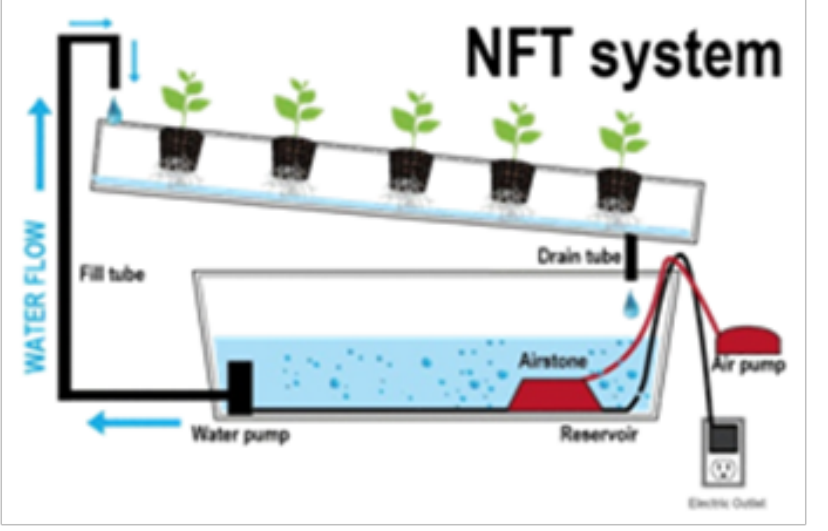

Figure 2 NFT systems.

\section{Simulation}

For the simulation process will be divided into four parts:
Register on the website/API: The user accesses the website and registers all the information about where it will make the automation, the environment name, the microcontroller and the things that will be connected to the microcontroller to be used.

Installation and Sign in middleware: By downloading the middleware executable file, the user will install and login, using the login information registered in the WEBSITE/API, the user will choose which microcontroller is connected to the computer, the same will play the role of coordinator.

Hardware connection: At this time will be the connection of the components to the microcontroller which is playing the role of coordinator. After all connected, implementation and compile code for the Arduino will be done.

Installation and use of the app: One of the ways of access to quick form of data will be the app where the user can download and install on your Smartphone. When installing, the user's login will be displayed where all the data of the things your automation will be done (Figure 3 ).

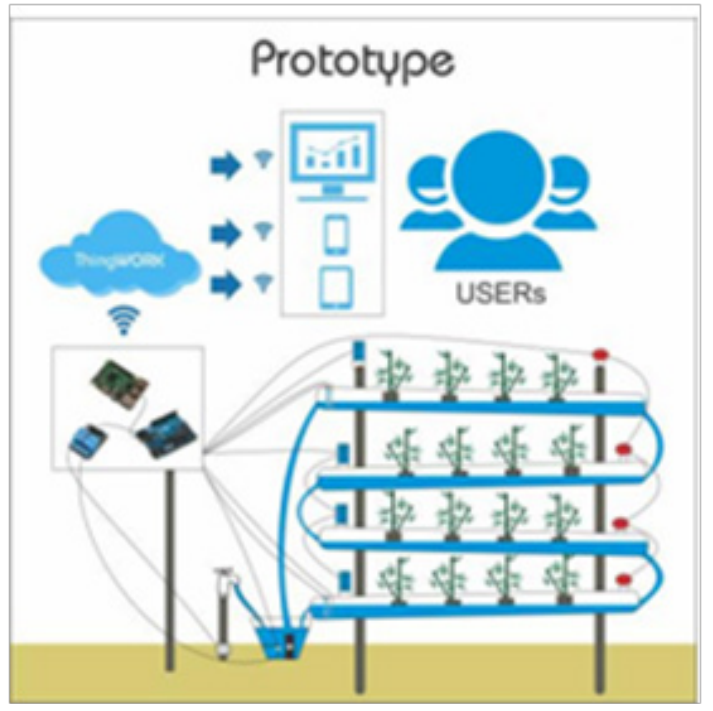

Figure 3 Prototype systems.

The whole system works only with the Internet, because the middleware needs to access the API that is hosted in the cloud. The app likewise needs the internet to access the data contained in the API. However, it was done to simulate an irrigation system using hydroponics and all automation Arduino as shown in Figure 4 being the coordinator and Raspberry Pi with the middleware; both acquired sensor data and were all part of water release valve and pump alone, without human intervention.

The whole process is observed by the user through the app and website, which in turn, would have access from anywhere in the world, and obtained all temperature control and humidity, ambient lighting, water temperature, water $\mathrm{pH}$ and the time at which the valve and pump were connected.

\section{Components}

Wi-Fi: or even the internet itself is the primary means of communication between the API that will be hosted in the cloud, mobile and middleware. 


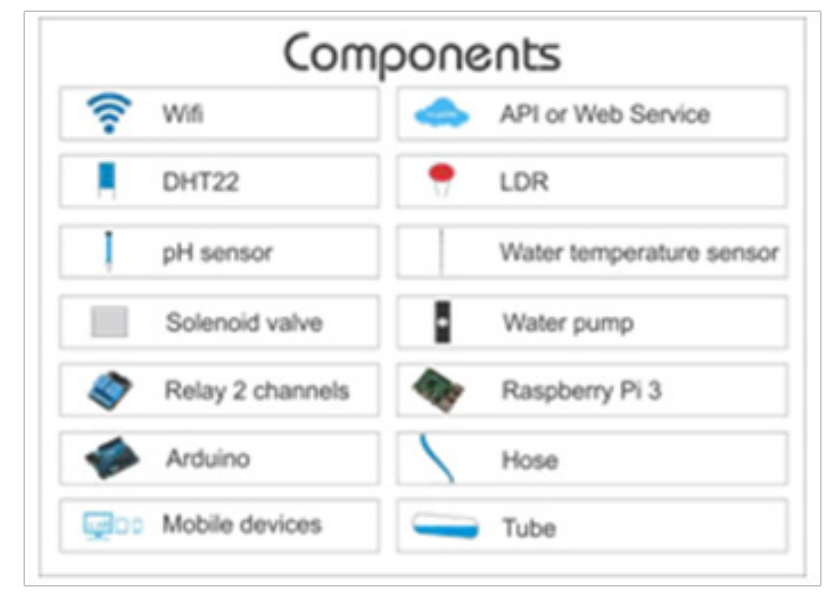

Figure 4 Components.

DHT22: Humidity Temperature Sensor air.

pH sensor: Sensor which will raise the $\mathrm{pH}$ of the water in analog form, as represented in shape having level from $0 \%$ to $100 \%$, as done via the programming code.

Solenoid valve: It is a valve that will be responsible for releasing or not the water to the faucet.

Raley 2 channels: Module which has 2 Raley. The raley is an electronic component that has the function to turn on and off other electronic components that work with voltages up to 220 volts. One is responsible for activating and deactivating the Solenoid valve and the other on and off the Water pump.

Arduino: It who will play the role of coordinator, the Arduino is a microcontroller that works with 5 volts and its operating voltage and has input ports and output, digital and analog. It also has a serial communication that allows communication between Arduino and computer or microprocessor via USB.

Mobile devices: Mobile devices: To access the information automation, you have two options, or access the website with your login and password, from any device, be it phone, computer, tablet, or others who have access to the Internet and web browsers like a browser. The second option would be through an app also implemented in this work, being a facilitator of information, where the user only needs to install on your phone or tablet with your login and password, thereby gaining access to all the components of your application.

API or Web Service: AA.P.I. (Application Programming Interface) or Web Service is a web application that can be implemented by any web language, but this work was implemented in Ruby language. It is the database containing all the information registered by the user, such as its features and services that will be available for the app and middleware. That is, the API and is the place where everything will center, will also serve as a communication bridge between devices and middleware, thus causing dristribuição and information exchange.

LDR: Light Dependent Resistor. An electronic component that will be used to measure the ambient light.

Water temperature sensor: sensor which will measure the water temperature.

Water pump: It will be responsible for playing the water to the top of hydroponic scheme. That is, it will be inside the bucket or tank, where it will play all the water in the scheduled time and the right time, as stated by the programmer in the code.

Raspberry Pi 3: Microprocessor that will play a middleware, it is only on the internet running a system that will be responsible for communication and exchange of data between the coordinator and the API. His choice was due to be the computer more affordable and less power consumption, thus making the most feasible cost-effective.

Hose: The hose will be higher as a pipe bridge, so that the pipes are beneath it, thereby carrying the water that is left for the next pipe.

Tube: For the construction of the prototype, pipes are used to make the entire structure to contain the plant and sensors. In most crops made with hydroponics scheme, pipes are used of the same type, thereby making the prototype here made closer to the end product.

\section{Conclusion}

With a monitoring and the environment that will be submitted to crops, can condition-ideally the same, thus obtaining benefits such as exemption from pesticide use; Increase the quality and production of vegetables; Cost-benefit in the medium and long term; Independence on site and climate, when grown on fully controlled media; As well as sustainability and ecology, as the drastic reduction of water use and allocation of space and soil, as in agriculture, not causing environmental impacts such as soil erosion, deforestation and others. One can also conclude that the automated system has great independence, but not consolidated in the case of hydroponic knowledge of art, as his agent analyzer makes decisions based on predefined rules.

\section{Future works}

As a proposal for future work, the prototype made this work will evolve to a larger scale, with the test closer application yet of the final product and showing its advantages and disadvantages as its application in small and large scale projects. The use of the End Device on a large scale would be a wireless interconnection way, thus making hydroponics systems connected via radio frequency or wifi. Seeing as the reference implementation of new technologies and covering increasingly automating the irrigation system shown in this work. In addition to developing the project for large-scale and connect them via the Internet, you have as future work the use of certain medications on time indicated by the sensing scheme itself, using artificial intelligence implemented or API or Arduino code itself. It is suggested to further research in this area, such as the proposal to implement a program of E, S (Expert System) with the agent analyzer, showing the model in order to ensure complete independence of an expert in hydroponics; And also implement a D.S. (Decision Support) based on Data Mining, aiding and optimizing even more quality, volume and production time and reducing costs.

\section{Acknowledgments}

None.

\section{Conflicts of interest}

The author declares there are no conflicts of interest.

\section{References}

1. CJ Graves. The Nutrient Film Technique. Horticultural Reviews, Janick J editor. USA, The AVI Publishing Company. 1983;5:1-44. 
2. Maansson LPS. Analisys-A Way to Register the Nutrient Uptake in Soilless Culture. 1984;339-346.

3. Resh HM. Hydroponic Food Production. Califórnia, Woodbridge Press Publishing Company. 1996;527.

4. Kevin Ashton. That internet of things thing. RFiD Journal 2009;22:97114.

5. Kortuem G, Kawsar F, Fitton D, et al. Smart objects as building blocks for the internet of things. Internet Computing, IEEE. 2010;14(1):44-51.

6. IoT-A, Internet of Things Architecture of the European Lighthouse Integrated Project. 2014.
7. Ferreira Hiro GC. Middleware Architecture for Internet of Things. Master's Dissertation in Electrical Engineering. 2014;42.

8. Menezes JR, Martins FOG, Fernandes SR. Growth and nutritional evaluation of lettuce grown in NFT with nutritive solutions of chemical and organic origin. Horticultura Brasileira. 2004;22(3):632-637.

9. Martinez HEP, JB Silva Filho. Introduction to Hydroponic Plant Cultivation. Viçosa MG. 1997;52.

10. Viana Juan Pablo, Frota Thyago Wesley, Veloso Artur Felipe da Silva. Automated Monitoring System applied to Hydroponic Cultures of Type NFT. 2015. 